\title{
Acculturation, economics and food insecurity among refugees resettled in the USA: a case study of West African refugees
}

\author{
Craig Hadley, ${ }^{1, *}$, Ariel Zodhiates ${ }^{2}$ and Daniel W Sellen ${ }^{3}$
}

${ }^{1}$ Center for Social Epidemiology \& Population Health, University of Michigan, Ann Arbor, MI 48104, USA: ${ }^{2}$ Brown University, Providence, RI, USA: ${ }^{3}$ Department of Anthropology, University of Toronto, Toronto, Ontario, Canada

Submitted 9 February 2006: Accepted 3 August 2006

\begin{abstract}
Objectives: To assess the occurrence and severity of food insecurity, and examine associations between food insecurity and measures of socio-economic status and indicators of acculturation.

Design: Structured interviews among a non-probability sample of West African refugees at baseline and 6 months' follow-up.

Setting: Mid-sized city in north-eastern USA.

Subjects: One hundred and one caregivers with children under the age of 5 years and who have been living the USA for fewer than 4 years.

Results: Food insecurity was indicated in approximately half of households (53\%). The occurrence of food insecurity was associated with measures of socio-economic status such as income, employment status and participation in the Food Stamp Program $(P<0.05)$. Measures of acculturation such as difficulty in the shopping environment and language difficulty were also associated with the occurrence and severity of food insecurity, as was time lived in the USA $(P<0.05)$. Among households who had been in the USA for 1 year or less, food insecurity was indicated in $73 \%$, whereas among households who had been in the USA for at least 3 years food insecurity was indicted in 33\%.

Conclusions: Refugees are a potentially vulnerable group and our results echo others in suggesting a need for more thorough monitoring of the health and well-being of recently resettled refugees. These results also suggest that reliance on employment as the sole indicator of success in the resettlement process may be too narrow an indicator. Future research should focus on a wider range of measures of health and well-being.
\end{abstract}

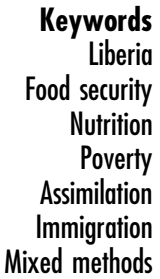

rords

security

Poverty

Assimilation

Mixed methods
There are currently 10 million individuals who meet the United Nations High Commissioner for Refugees' definition of a refugee. These are individuals who are unable to return to their country of origin because of a wellgrounded fear of persecution. A majority of these individuals will be settled into neighbouring countries and many will ultimately be repatriated. However, each year approximately 100000 individuals will seek a more durable solution and will be resettled into a developed country, with the USA accepting up to 70000 individuals per year. Increasingly, the resettled population is being dominated by African refugees, with 29000 individuals being resettled in 2004 alone, and the number of complex humanitarian emergencies in Africa promises to produce more refugees over the coming years. Although the food insecurity situation is described well for refugee camps ${ }^{1}$, little is known about the food security situation of refugees once they have been resettled in the USA, where most research on these groups has focused on health at the time of entry $^{2}$ or mental health outcomes ${ }^{3}$. This is unfortunate as secure access to sufficient food at all times is a basic human right and an important dimension of individual and family well-being. In developed country settings, where its occurrence has been linked with a range of poor health outcomes and poor dietary practice, food insecurity may serve as a key indicator of one aspect of the transition into life in the USA and provide insight into the period following initial resettlement which has not yet received much attention.

The objectives of the present study were to use an exploratory ethnographic approach to examine food insecurity and its manifestations among members of a resettled West African refugee community and then to assess the magnitude and severity of food insecurity using a survey of West African refugees living in a mid-sized US city. Secondary objectives were to examine the relationships between socio-economic status and indicators of acculturation and household food insecurity. 
Food security exists when people have predictable access at all times to nutritious and culturally appropriate foods. Food insecurity occurs whenever appropriate and safe foods are not available or the ability to acquire such foods is limited or uncertain ${ }^{4}$. Conceptually, food insecurity is a more direct measure of inadequate or unreliable dietary supply than is low income because it more closely taps into the phenomena of interest ${ }^{5}$. Food insecurity represents a major public health concern and is a useful index of health and well-being because it is associated with poverty, ill health, poor dietary intake (e.g. low intake of fruits and vegetables), limited social capital, depressive disorders and, paradoxically, overweight and obesity among females ${ }^{6-10}$. Given the links between food insecurity and negative health, behavioural and schooling outcomes, food insecurity may serve as a barrier to successful integration in US society, an occurrence that produces health inequalities, and a useful marker of overall health and well-being among this vulnerable subpopulation.

In the USA, where approximately $11 \%$ of households are food-insecure, food insecurity and its more severe form hunger - ultimately results from insufficient income ${ }^{11}$. The income-food insecurity relationship may be modified by differences in shopping practices, food preferences and budget management. Refugees resettled into the USA and other developed countries are hypothesised to be at high risk of food insecurity because upon arrival they face high levels of unemployment and often have low incomes. Dietary acculturation, which is both the outcome and the process through which immigrants acquire and react to food-related habits of the dominant society ${ }^{12}$, might also be linked to the occurrence and severity of food insecurity, and this might occur through multiple pathways. For instance, newcomers from developing countries are likely to face tremendous shifts in budget management and diet and food-related practices (e.g. shopping, preparation $)^{13,14}$. Home budget management may, for example, predispose to early completion of income, either before the next pay cheque or before the next food stamp cycle begins ${ }^{15}$. Food shoppers may seek out food items commonly consumed in their country of origin, the prices of which may not be compatible with their current monetary situation ${ }^{16,17}$. These difficulties may be coupled with and exacerbated by language barriers, which have been shown to be associated with food insecurity among other low-income populations ${ }^{9,18}$. Thus, measures of socio-economics as well indicators of acculturation, such as difficulty in the new shopping environment and language difficulties, may contribute to food insecurity. In line with this expectation, pilot evidence from this population and other refugee populations suggests that food insecurity is a potential public health concern, and one that is related to social, economic and cultural factors $^{13,19}$. However, we are aware of no other studies of food insecurity among Liberian refugees living in the USA.
On the basis of this short review, we hypothesised that food insecurity among this convenience sample of resettled African refugees would be associated with measures of acculturation and socio-economics. Specifically, we hypothesised that food insecurity would be greater among women who reported (1) lower income and education, and related to several measures of acculturation including (2) a shorter duration in the USA, (3) difficulties in understanding others and (4) greater reported difficulties in the food-related environment.

\section{Methods}

Our qualitative work consisted of participant observation in several health meetings, discussions with a nurse and social worker who were active in the community, informal interviews with refugees, and 15 in-depth interviews with women who met the inclusion criteria for the study. The in-depth interviews followed a topic guide that touched on issues of food insecurity, dietary acculturation, food preparation and difficulties in the USA. Following several months of this ethnographic work, informal interviews and a pilot study ${ }^{19}$, a service-based convenience sample was used to enrol West African women into the study. All women (hereafter called caregivers) who met the following inclusion criteria were eligible for the study: 18 years or older, has a child under 5 years of age, living in the USA for less than 4 years, and claimed Liberia as country of birth. These women were recruited through the primary resettlement agency, meeting points of the Women, Infants, and Children Program (WIC; a public assistance programme with set income criteria), church groups and snowball sampling methods. All caregivers gave their informed consent and all procedures were approved by Brown University.

Study participants were interviewed at their homes in English (the national language of Liberia) by female West African interviewers. The interview was conducted using a standardised data collection instrument that collected information on migration history, current household composition and economics, participation in food assistance programmes, perceived difficulty with shopping and language, and, at a 6-month follow-up, household food insecurity in the previous six months. The interviewers had undergone extensive training in a pilot phase of the project $^{19}$, through role playing and several pre-tests. A food insecurity instrument was created based on the US Department of Agriculture (USDA) food insecurity scale, and was employed to measure the food security of the respondent's household over the course of the previous six months ${ }^{4}$. The primary modifications to the instrument included changing the item related to 'balanced' foods to read 'have meals with many different foods, like meat, fish, fruits and vegetables', replacing the word 'afford' with 'because you didn't have enough money', and removal of three questions regarding the frequency of occurrence of 
food insecurity. The statements were also asked as questions ${ }^{20}$. For these reasons results from this study are not directly comparable to those from other studies using the USDA tool, but they are likely to be similar. These modifications were made to ensure that the instrument was culturally appropriate and to reduce redundancy and respondent burden. Caregivers were presented with 15 statements about the household's food security situation that varied in their severity of the food insecurity situation depicted (Table 2). On the basis of a caregiver's response patterns (each affirmative answer scored 1), households were categorised as food-secure $(<3$ affirmative responses) or experiencing mild food insecurity (3-7 affirmative responses) or severe food insecurity $(>7$ affirmative responses). A household's food security situation was also measured using a continuous measure computed by summing the number of affirmative responses to the scale. This latter measure is perhaps a more accurate measure of food insecurity because it provides more information about the severity of the food insecurity situation ${ }^{11}$. Modifying existing instruments potentially jeopardises their psychometric properties, and we did not formally validate our instrument. Nevertheless, a large number of studies using similar scales and items strongly suggest that the 'core' experience of food insecurity is similar across a broad array of populations, suggesting that instruments that tap this core, like ours, are likely to be valid ${ }^{21}$. Additionally, our prior work and formative work also suggested that the instrument has face validity. Food insecurity experienced prior to arrival in the USA was assessed with a single question which asked the caregivers whether they ever ran out of food before having money to buy more prior to relocating to the USA.

Several socio-economic and cultural variables were measured as well. A scale representing the process of dietary acculturation was constructed according to each caregiver's response to six statements about shopping. The items comprising the scale were derived directly from qualitative work that occurred prior to initiation of the survey and tapped several different elements of the dietary acculturation process, including statements such as 'I cook African foods because I don't know how to cook anything else', 'I shop where I do because I don't know where other food stores are' and 'It is difficult for me to shop because I don't know what all the different foods [in the store] are'. Responses were scored as agreed or disagreed and internal consistency of the dietary acculturation scale was adequate (Cronbach's $\alpha=0.73$ ), so the items were summed to create an aggregate score. Other measures of acculturation included self-reported language ability, which was assessed by asking caregivers whether they currently had any difficulty understanding people in the USA because of language, and the time in months since arrival in the USA. Caregivers were also asked to estimate their own monthly income, whether they were currently employed and whether they were participating in the federal Food Stamp Program (FSP; a public assistance programme with set income criteria for eligibility), all measures of socio-economic status. At the 6-month followup, women were also asked if they ever finished their food stamps before being able to get more; a risk factor for food insecurity in other studies ${ }^{15}$.

\section{Analyses}

For the qualitative data, transcripts were entered into a qualitative data analysis software program, and key themes and illustrative quotes were collected. Details relevant to dietary acculturation were also extracted and were integrated into the study questionnaire. All data analysis was carried out using SPSS $^{22}$ and the criterion for statistical significance was set at 0.05 . Descriptive statistics were compared between groups using the chi-square test and the Wilcoxon two-sample test. Associations between continuous and ordinal variables (such as time in the USA and the food security scale) were examined using the Spearman test. Finally, in an attempt to identify independent predictors of food insecurity, several variables identified as significant predictors of the severity of food insecurity were entered into a multivariate regression model. Because several of the acculturation variables are conceptually similar, collinearity was problematic in the regression models. To mitigate this, we used principal components analysis to create a single variable that represented acculturation. This was created from the dietary acculturation scale, time lived in the USA and the language difficulty item, and resulted in a single component that accounted for about $60 \%$ of the variance. This variable was used in the multivariate model described below.

\section{Results}

\section{Formative research}

Here we highlight three themes that emerged from the qualitative work: the causes of food insecurity, coping mechanisms and the consequences of food insecurity.

In the USA, food insecurity has its roots in insufficient economic resources, and limited resources force individuals to make allocation decisions. Such decisions were clear from the participants' statements and although they took many forms, the statements all highlighted the difficult tradeoffs that families had to make. One participant expressed this well:

\section{'...if you're working, it means they have to take your cash benefit from you, but they can't take your food stamps. Because if they take your food stamps, it cannot afford your rent. Because you have to focus on your rent, not to put you outside, and you have to focus on your car, and it's a big hell of a problem because you have to eat.'}

Others reflected on this same issue by calling attention to their large utility bills and contrasting these with their family's comparatively small incomes. This theme of 'small 
money-large bills' replayed itself several times. As one respondent put it:

'Ob yeah, I came with my busband, and he working, and one day, one day per week, one day sometimes $\$ 40$ check, and I have to say, what can I do with $\$ 40$ check? In America we get small money and have to pay your bills, your rent, everything.'

'Bills' was nominated by $33 \%$ of the women as the most difficult feature of living in the USA, and caseworkers noted frequently the difficulty that some newcomers had with payment and budget management.

A unique feature of the refugee situation that might impact food insecurity also emerged as potentially important. Obligations to family both in West Africa and in the USA were cited by several respondents as a significant strain on familial resources. Family who were not yet able to be resettled in the USA frequently phoned to ask that money be sent back home. Further, upon arrival and before an individual was 'in the system', newly arrived refugees would rely on those family members who had already been living in the USA for some time. These two themes are illustrated in these two quotes:

Participant 1: 'I have to belp when my mother calls, because my mother she has eight children, and they are little children in Africa, and I was the firstborn for my mom. So they call and say, "A dog bit your little brother and they take the flesh off," and the money I was having, I send it on the same spot. So no food...'

Participant 2: 'When her sister first came [to the USA], and her sister didn't get food stamps, so they were sharing the food stamps, and she was worried, like how would I get enough food for everyone in the house?'

As a consequence of food and financial insecurity, many caregivers reported altering their behaviour or attempting to alter their children's behaviours especially as they related to food and finances. These responses included shopping at different food stores, purchasing fewer or different food items, reducing the amounts consumed, and attempting to limit consumption and waste. One participant commented that in the face of financial difficulty she

'...decided to go to the cheaper place and buy more food instead of going to the expensive place.'

Another participant altered the amount of food she used while cooking:

‘... [instead of using] two cups over here, I cook one and a balf, just so it can reach.'

This exploratory phase along with our previous pilot work suggested that issues related to food and difficulties were able to be openly discussed and that food insecurity might be an important public health issue in this
Table 1 Selected survey sample characteristics $(n=101)$

\begin{tabular}{lc}
\hline Variable & Measure \\
\hline Household size & $5.3(2.2)$ \\
Household income $<\$ 1000$ per month & 51 \\
Respondent's income $<\$ 1000$ per month & 64 \\
Food Stamp Program - ever & 98 \\
Food Stamp Program - currently & 48 \\
Women, Infants, and Children & 59 \\
$\quad$ Program - currently & $(9.4), \min =18$, \\
Age (years) & max $=74$ \\
High school education or higher & 57 \\
Employed at baseline & 57 \\
Self-perceived difficulty with English & 38 \\
at baseline & $2002(1.19)$ \\
Year arrived in the USA & $22.1(14.2)$ \\
Months in the USA &
\end{tabular}

Data are presented as mean (standard deviation) (plus minimum and maximum for age) or frequency (\%).

community. This helped guide our instrument design and provided insight into risk factors for food insecurity.

\section{Survey}

The final sample size for the survey was 101 caregivers and there were no refusals (Table 1). At baseline mean household size was five individuals, one or two of whom were under the age of 5 years. Caregivers were on average 30 years of age with a range from 18 to 74 years. The women had been in the USA for an average of 22 months (standard deviation (SD) 14.2). Just over half of the caregivers (57\%) interviewed had a high school education or higher, and $57 \%$ were currently employed. Half of the caregivers reported mean household income as less than $\$ 1000$ per month, and $64 \%$ of caregivers reported their own income as less than $\$ 1000$ per month. Nearly all caregivers had participated in the FSP at some point since their arrival (98\%), and approximately $48 \%$ of caregivers were currently participants. Of those women participating in the FSP, 52\% completed their stamps 'early'. Just over half (59\%) of the caregivers were currently were participating in WIC, and slightly more than one in three (38\%) reported experiencing some difficulty understanding people in the USA. Participation in the FSP $(P<0.0001)$ and unemployment $(P=0.001)$ were negatively related to time lived in the USA. Individual items from the dietary acculturation scale were generally associated with time in the USA and with difficulties in understanding people because of language (all $P<0.05$ except for two items: one's preference/ perceived difficulty in shopping in large supermarket-type stores were not significantly associated with time in the USA or language difficulties).

Approximately 53\% of caregivers' responses indicated that they and members of their households had experienced periods of food insecurity during the six months prior to the follow-up interview. This 53\% comprised $37 \%$ who experienced mild food insecurity and $16 \%$ whose responses to the food insecurity scale 
indicated severe food insecurity. The mean food insecurity score on the continuous scale, indicating severity, was 3.6 (SD 3.4). The food security scale showed acceptable internal consistency (Cronbach's $\alpha=0.85$ ) and affirmative responses fit with expectations in that more respondents agreed with the less severe statements. For example, 60\% of respondents stated that they worried about their food supply while no respondent reported that their children had to miss food for the whole day (Table 2). A majority of caregivers responded that they had experienced food insecurity prior to arriving in the USA (90\%).

In bivariate relationships, the occurrence of food insecurity and its severity were related to several economic variables (Table 3). Food-insecure households were more likely to have a reported monthly income of less than $\$ 1000(P=0.013)$ and to have primary caregivers who were less likely to be employed $(P=0.035)$. Caregiver education was also associated with food security: women with a high school education or higher were less likely to be food-insecure $(P=0.039)$. Food-insecure households were also more likely to be participating in the FSP $(P=0.033)$ and the WIC $(P=0.040)$ and experienced more severe food insecurity. Among FSP participants, 52\% reported completing their food stamps before they were able to get more, and these individuals were far more likely to be food-insecure $(P<0.0001)$. There was no relationship between a caregiver's 6-month food insecurity situation and ever having received food from a food bank or church $(P=0.29)$. Scores on the food insecurity scale were positively related to household size $(P=0.01)$ but not caregiver age $(P=0.54)$.

The occurrence of food insecurity and the severity of a household's food security situation were also associated with indicators of acculturation (Table 3 ). Those caregivers who reported experiencing difficulty understanding people because of language problems were somewhat more likely to be food-insecure $(P=0.054)$ and experienced more severe food insecurity $(P=0.021)$.
Higher scores on the dietary acculturation scale, indicating lower dietary acculturation, were also associated with food insecurity $(P=0.038)$ and the severity of food insecurity $(P=0.009)$. Finally and encouragingly, time lived in the USA was strongly and negatively associated with food insecurity $(P<0.001)$ and severity $(P<0.0001)$. Among households who had been in the USA for 1 year or less, food insecurity was indicated in 73\%, whereas among households who had been in the USA for at least 3 years, food insecurity was indicted in only 33\% (Fig. 1).

In the multivariate model, first we entered the score on the dietary acculturation scale, whether the respondent was working at baseline, language difficulty and months in the USA as independent variables. In this model, only the variable time in the USA was significant and only marginally so $(P=0.054)$, yet the overall model was highly significant $\left(F=4.6, P=0.002, R^{2}=16 \%\right)$. Low $P$-values for individual covariates but a high $P$-value on the final model is indicative of collinearity. The second method used was to enter the acculturation factor from the principal components analysis (which explained more than $60 \%$ of the variance) along with three different proxy measures of income: whether the respondent reported a household income of less than $\$ 1000$ per month, whether the respondent was employed at baseline and whether the respondent was participating in the FSP, which has an income eligibility requirement. In each of these models, the acculturation variable was significant $(P=0.02)$ while tests for significance for the income variables were nonsignificant $(P \gg 0.05)$. Household size was also a positive and independent predictor of food insecurity in each model $(P=0.04)$.

\section{Discussion}

To our knowledge, this is one of the largest communitybased studies of the public health nutrition of African refugees resettled in the USA. The key result from this study

Table 2 Items on the food insecurity scale and proportion of affirmative responses

\begin{tabular}{l}
\hline During the last six months... \\
\hline 1. Were you ever worried whether your food would run out before you got money to buy more? \\
2. Did you run out of food before having money to get more? \\
3. Did you run out of money to have meals with many different foods, like meat, fish, and fruits and vegetables? \\
4. Did you have to buy only a few kinds of inexpensive foods to feed your children because you ran out of money? \\
5. Were you not able to give your children a meal that included many different foods, like meat, fish, and fruits and \\
vegetables, because you didn't have enough money? \\
6. Did any of your children ever not eat enough because there was not enough money? \\
7. Did you or other big people in your house ever cut or reduce the size of meals because there wasn't enough \\
money to buy food? \\
8. Did you ever eat less than you felt you should because there wasn't enough money to buy food? \\
9. Were you ever hungry but did not eat because there wasn't enough money to buy food? \\
10. Have you lost weight because you didn't have enough money for food? \\
11. Did you ever not eat for a whole day because there wasn't enough money for food? \\
12. Did you ever cut or reduce the size of your children's meals because there wasn't enough money for food? \\
13. Did any of the children ever skip meals because there wasn't enough money for food? \\
14. Were your children ever hungry because there wasn't enough money for food? \\
15. Did your children ever not eat for a whole day because there wasn't enough money for food? \\
\hline
\end{tabular}


Table 3 Bivariate tests comparing proportion of food-secure and food-insecure households by selected covariates

\begin{tabular}{lccc}
\hline & Food-secure & Food-insecure & $P$-value* \\
\hline Language difficulties (\% yes) & 34 & 66 & 0.05 \\
Employed (\% no) & 31 & 68 & 0.04 \\
High school education (\% no) & 34 & 66 & 0.04 \\
Dietary acculturation (\% low)† & 39 & 61 & 0.01 \\
In USA for <12 months (\% yes) & 27 & 72 & 0.007 \\
Earns less than \$1000 per month (\% yes) & 37 & 63 & 0.01 \\
\hline
\end{tabular}

${ }^{*}$ Chi-square test.

† Dietary acculturation index split at median, $P$-value differs from text.

is that food insecurity was associated with length of stay in the USA: refugees initially face high levels of food insecurity, particularly during the first year, and the prevalence declines thereafter. Importantly, however, one-third of households still showed evidence of food insecurity after 3 years in the USA, for reasons that are not yet clear. Bivariate results also indicated that food insecurity was associated with multiple measures of socio-economic status, participation in the FSP and employment status. The results presented here also support hypotheses that food insecurity is related to acculturation status, possibly operating through the management of resources and difficulty in the food shopping environment. These results suggest that programmes to expedite the transition from unemployed to employed status are needed and may have a substantial impact on the food security situation among female caregivers with young children. Additionally, they suggest that programmes at the resettlement centre that seek to improve knowledge of budgeting and shopping practices may also prove beneficial in reducing the likelihood of food anxiety or food deprivation.

The inferences drawn from this work need to be taken with caution given several limitations of the study design. First, the associations between time in the USA and outcomes may not necessarily reflect causal relationships. Second, without having access to the full list of newly arrived refugees, we cannot know how representative this sample is of the wider refugee population. Although it is

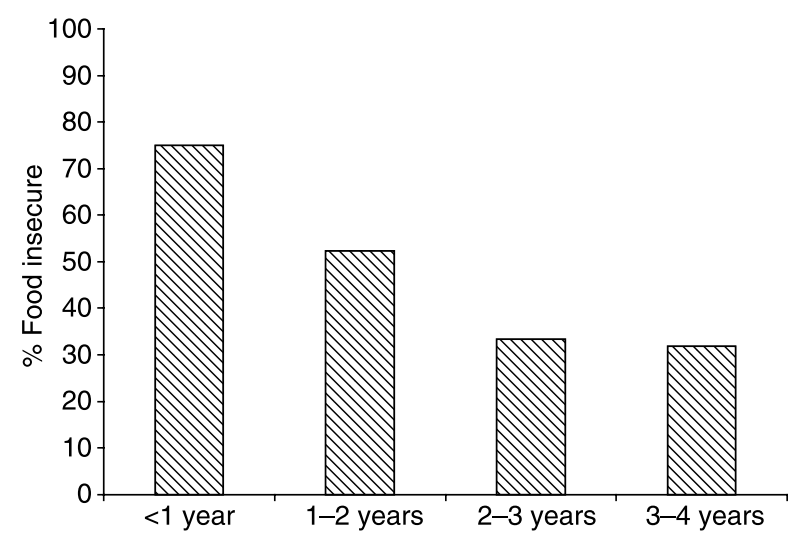

Fig. 1 Occurrence of food insecurity by time (in years) respondent has lived in the USA $(n=101)$ encouraging that no one refused to participate in the study, it is possible (albeit unlikely) that there exist some segments of the population that were not reached by our interviewing team. Nevertheless, our focus on women and children reflects the fact that, in $2004,55 \%$ of Liberian newcomers were female, $60 \%$ were adults and $50 \%$ were 17 years of age and younger. Thus, our focus on women with children matches the general age and sex distribution of the population, although we may have under-sampled women with children over the age of 5 years ${ }^{23}$. Regardless of how representative our sample is of the wider Liberian refugee population, the relationships we identified between variables are important and generally correspond with our theoretical predictions and ethnographic evidence. Third, although the multivariate models suggest acculturation drives food insecurity more so than does income, we acknowledge that measuring each of these variables is difficult, especially the construct of acculturation. As we were particularly interested in dietary acculturation, and more specifically in the mechanisms that lead to dietary acculturation, we did not focus on measuring other dimensions of acculturation, including acculturation that may have occurred prior to arrival in the USA. Individuals who lived in large urban centres prior to settling in the USA may have much less difficulty adapting to the food-related ecology in the USA; future research should seek to measure the pre-departure acculturation status as this may account for substantial post-migration change. A strength of the study is the qualitative work that helped us overcome some of the difficulties in measuring acculturation or, more appropriately, the pathways that lead to acculturation. This stage was utilised in part to generate ideas about the relevant dimensions of dietary acculturation, which was subsequently included on our survey instrument.

The results of this work are in broad agreement with similar but smaller studies of refugees and other immigrants to the USA. For instance, our work with this population during a pilot phase revealed high levels of food insecurity and a similar set of risk factors ${ }^{19}$. That study $(n=33)$ actually revealed considerably higher levels of food insecurity (85\%) and child hunger (45\%). Differences in study design, study instrument and location of interviews limit our ability to directly compare the results. For instance, in our pilot we used the Radimer/Cornell scale which may 
have influenced responses and subsequent conclusions. Perhaps more importantly, nearly all women were recruited from and interviewed at the local resettlement agency. This may have introduced context effects and biased our selection of respondents to those who were most in need and who were therefore spending more time at the settlement centre. Sellen et al. (unpublished) carried out a study with 60 recently resettled Sudanese refugees living in Atlanta, Georgia. That study indicated that nearly $75 \%$ of caregivers experienced food insecurity during their initial year in the USA. However, because women were not included after 1 year we do not know if levels of food insecurity declined with increasing amount of time in the USA. A study of 139 African refugees and migrants living in Australia, however, reported no cases of food insecurity, although food insecurity was assessed with a single item that represented extreme food insecurity ${ }^{24}$. Nevertheless, these studies suggest that refugees may be facing periods of concern over their food supply and food deprivation, which may in turn influence other health outcomes. Importantly too, food insecurity and food insufficiency have been associated with maternal depression in a number of studies ${ }^{25}$. Thus, refugees may initially face multiple burdens on their mental health.

The finding that those caregivers who completed their food stamps earlier in the cycle were far more likely to be food-insecure is an important one. This relationship suggests that management strategies might be an important area for intervention and education, as has been suggested for other low-income populations. For instance, Perez-Escamilla et al. ${ }^{15}$ showed in a communitybased study of low-income FSP participants in Hartford, Connecticut that early completion of food stamps was associated with increased likelihood of being food-insecure. Caseworkers at resettlement agencies have also reported that paying bills and managing money are among the primary difficulties experienced by newcomers to the USA. Regardless, formal assistance, such as the federal FSP, offers an important safety net for refugees and many women noted in informal interviews that such programmes were critical. The finding that participation in the FSP was predictive of food insecurity is likely an example of reverse causation: these households are likely poorer, which influences both food insecurity and their eligibility for public assistance.

Our results also indicate a negative association between FSP use and time in the USA, which might indicate that longer periods of time in the USA are causally related to increased financial security, a result in accordance with the report of Bollinger and Hagstrom ${ }^{26}$. While it is encouraging that the data lend support to the hypothesis that greater amounts of time living in the USA are associated with reduced likelihood of participating in the FSP, the finding that food insecurity still exists among non-FSP participants who have lived in the USA for more than 2 years is potentially important. This result is consistent with literature documenting the impact of moving off welfare (see for instance Cancian ${ }^{27}$ ). Anecdotal evidence from participants in our study about food insecurity resulting from lost benefits once mothers began working is supported by research performed in six cities across the USA looking at welfare reform and the health of young children $^{28}$. Cook et al. found that, even after controlling for potential confounding factors, children in families whose welfare was terminated or reduced, either by sanctions or administratively due to changes in income or expenses, were more likely to be food-insecure than children from households still receiving benefits ${ }^{28}$. This finding might suggest that employment, in and of itself, should not be seen as an endpoint for refugee resettlement programmes.

\section{Conclusions}

The results of this work, and others, suggest that African refugees who have sought resettlement in the USA are successfully managing at least some of the many challenges that come with relocation into a Westernised country. For instance, it is encouraging that those caregivers who had been in the USA for longer amounts of time were far less likely to be participants in the FSP, especially in light of the fact that some measures of immigrant health often show declines with increasing amounts of time in the USA ${ }^{29,30}$. Also encouraging were the clear associations between length of time in the USA and the likelihood that a caregiver was employed. It is discouraging, however, that nearly one of three caregivers indicated food insecurity even after having lived in the USA for more than 2 years. This is well past the point at which refugees are considered selfsufficient. Finally, the results suggest that acculturation but not proxy measures of income drive the food insecurity decline. Identifying the barriers to food security after extended stays in the USA will be an important next research step, as will investigating the potential impact of food insecurity on other domains of health and well-being.

\section{Acknowledgements}

The authors gratefully acknowledge the assistance of Alice Howard, Rebecca Dine, Julia Makor, Diane Rodriguez, Heather Ross, and the dedicated and extremely hardworking staff at the International Institute. Funding for this project came from the USDA Economic Research Service Small Grants Program. C. H. is a Robert Wood Johnson Health and Society Scholar. He thanks the Robert Wood Johnson Foundation's Health and society program for its financial support.

\section{References}

1 Toole MJ, Waldman RJ. The public health aspects of complex emergencies and refugee situations. Annual Review of Public Health 1997; 18: 283-312. 
2 Maroushek SR, Aguilar EF, Stauffer W, Abd-Alla MD. Malaria among refugee children at arrival in the United States. Pediatric Infectious Disease Journal 2005; 24: 450-2.

3 Fazel M, Wheeler J, Danesh J. Prevalence of serious mental disorder in 7000 refugees resettled in Western countries: a systematic review. Lancet 2005; 365: 1309-14.

4 Bickel G, Nord M, Price C, Hamilton W, Cook J. Guide to Measuring Food Insecurity, revised 2000. Alexandria, VA: US Department of Agriculture, Food and Nutrition Service, March 2000.

5 Frongillo EA. Validation of measures of food insecurity and hunger. Journal of Nutrition 1999; 129: 506S-9S.

6 Alaimo K, Olson CM, Frongillo EA Jr. Low family income and food insufficiency in relation to overweight in US children: is there a paradox? Archives of Pediatrics \& Adolescent Medicine 2001; 155: 1161-7.

7 Alaimo K, Olson CM, Frongillo EA. Family food insufficiency, but not low family income, is positively associated with dysthymia and suicide symptoms in adolescents. Journal of Nutrition 2002; 132: 719-25.

8 Cook JT, Frank DA, Berkowitz C, Black MM, Casey PH, Cutts $\mathrm{DB}$, et al. Food insecurity is associated with adverse health outcomes among human infants and toddlers. Journal of Nutrition 2004; 134: 1432-8.

9 Himmelgreen D, Pérez-Escamilla R, Segura-Millán P, Gonzalez A, Singer M, Ferris A. Food insecurity among low-income Hispanics in Hartford, Connecticut: implications for public health policy. Human Organization 2000; 59: $334-42$.

10 Townsend MS, Peerson J, Love B, Achterberg C, Murphy SP. Food insecurity is positively related to overweight in women. Journal of Nutrition 2001; 131: 1738-45.

11 Nord M, Andrews M, Carlson S. Household Food Security in the United States. Research Report No. 35. Washington, DC: Food Assistance and Nutrition Research Program, Food and Rural Economics Division, Economic Research Service, US Department of Agriculture, 2002.

12 Himmelgreen D, Bretnall A, Perez-Escamilla R, Peng Y, Bermudez A. Birthplace, length of time in the US, and language are associated with diet among inner-city Puerto Rican women. Ecology of Food and Nutrition 2005; 44: $104-22$.

13 Sellen D, Tedstone A. Assessing food insecurity and nutritional well-being of preschool refugee children in the UK. In: Kerhsen J, ed. Food in the Migrant Experience. Burlington, Vermont: Ashgate, 2002; 214-28.

14 Hersey J, Anliker J, Miller C, Mullis RM, Daugherty S, Das S, et al. Food shopping practices are associated with dietary quality in low-income households. Journal of Nutrition Education and Behavior 2001; 33: S16-25.

15 Perez-Escamilla R, Ferris AM, Drake L, Haldeman L, Peranick J, Campbell M, et al. Food stamps are associated with food security and dietary intake of inner-city preschoolers from
Hartford, Connecticut. Journal of Nutrition 2000; 130: $2711-7$.

16 Jetter K, Cassady D. The availability and cost of healthier food items. Agricultural Issues Center Issues Brief 2005; 29.

17 Drewnowski A, Specter S. Poverty and obesity: the role of energy density and energy costs. American Journal of Clinical Nutrition 2004; 79: 6-16.

18 Kaiser LL, Melgar-Quinonez HR, Lamp CL, Johns MC, Sutherlin $\mathrm{JM}$, Harwood JO. Food security and nutritional outcomes of preschool-age Mexican-American children. Journal of the American Dietetic Association 2002; 102: 924-9.

19 Hadley C, Sellen D. Food insecurity and child hunger among recently resettled Liberian refugees and asylum seekers: a pilot study. Journal of Immigrant and Minority Health 2006; S: 369-75.

20 Perez-Escamilla R, Segall-Correa A, Kurdian Maranha L, Sampaio M, Marin-Leon L, Panigassi G. An adapted version of the US Department of Agriculture food insecurity module is a valid tool for assessing household food insecurity in Campinas, Brazil. Journal of Nutrition 2004; 134: 1923-8.

21 Coates J, Frongillo E, Rogers B, Webb P, Wilde P, Houser R. Commonalities in the experience of household food insecurity across cultures: what are measures missing? Journal of Nutrition 2006; 136: 1438S-48S.

22 SPSS Inc. SPSS for Windows, Release 11.0.1. Chicago, IL: SPSS Inc., 2001.

23 Department of State. Proposed Refugee Admissions for Fiscal Year 2005: Report to the Congress. Washington, DC: Department of State, 2005.

24 Renzaho R, Burns C. Post-migration food habits of subSaharan African migrants in Victoria: a cross-sectional study. Nutrition and Dietetics 2006; 63: 91-102.

25 Siefert K, Heflin CM, Corcoran ME, Williams DR. Food insufficiency and physical and mental health in a longitudinal survey of welfare recipients. Journal of Health and Social Behavior 2004; 45: 171-86.

26 Bollinger C, Hagstrom P. Food stamp participation of refugees and immigrants [online], 2003. Available at http://www.ukcpr.org/Publications/fs_refugee3.3.pdf. Accessed 7 March 2004.

27 Cancian M. Rhetoric and reality of work-based welfare reform. Social Work 2001; 46: 309-14.

28 Cook JT, Frank DA, Berkowitz C, Black MM, Casey PH, Cutts DB, et al. Welfare reform and the health of young children: a sentinel survey in 6 US cities. Archives of Pediatrics \& Adolescent Medicine 2002; 156: 678-84.

29 Goel MS, McCarthy EP, Phillips RS, Wee CC. Obesity among US immigrant subgroups by duration of residence. Journal of American Medical Association 2004; 292: 2860-7.

30 Kandula NR, Kersey M, Lurie N. Assuring the health of immigrants: what the leading health indicators tell us. Annual Review of Public Health 2004; 25: 357-76. 